\title{
Pain Management for Traumatic Rib Fractures with ESP Block in ICU
}

\author{
Canan Gursoy1, Yagmur Kuscu² and Semra Gumus Demirbilek² \\ 1Division of Intensive Care Unit, Department of Anesthesiology and Reanimation, Mugla Sitki Koçman University Training and \\ Research Hospital, Mugla, Turkey \\ 2Department of Anesthesiology and Reanimation, Mugla Sitki Koçman University, Mugla, Turkey
}

\begin{abstract}
Rib fractures are common injuries in blunt chest trauma, that cause severe thoracic pain, which limits patients' ability to cough and breathe deeply, which can lead to atelectasis and pneumonia. Various treatments for pain management of rib fractures have been described such as analgesics and regional anaesthesia. The ultrasound-guided erector spinae plane block (ESP) is a novel myofascial plane block for thoracic analgesia after thoracic or abdomen surgery. It is simple to perform the block because the key landmarks of tip of transverse processes and erector spinae muscle are easily visualised on ultrasound. This also allows to treat patients more easily in intensive care unit (ICU). We present a case that used ESP for pain management related to traumatic multiple rib fractures and prevented pulmonary complications with ESP block in ICU.
\end{abstract}

Key Words: Analgesia, Erector spine plane block, Rib fractures

How to cite this article: Gürsoy C, Kuscu Y, Demirbilek SG. Pain management for traumatic rib fractures with ESP block in ICU. J Coll Physicians Surg Pak 2020; 30(3):318-320.

\section{INTRODUCTION}

Rib fractures are common injuries in blunt chest trauma. These are clinically important because they can cause severe thoracic pain. ${ }^{1}$ The thoracic pain limits patients' ability to cough and breathe deeply, which can lead to atelectasis and pneumonia. ${ }^{2}$ At the end, the patient requires prolonged mechanical ventilation and stay in hospital and intensive care unit (ICU) owing to secondary pulmonary complications.

Various treatments for pain management have been described in the literature, such as oral or parenteral analgesics, intercostal nerve blocks, intrapleural catheters, epidural analgesia; and thoracic paravertebral block. ${ }^{1}$ According to meta-analysis by Peek et al., regional anaesthesia is superior to oral and parenteral analgesia. ${ }^{2}$ Ultrasound-guided erector spinae plane block (ESP) is a novel myofascial plane block recently utilised in clinical practice for pain management after rib fractures and surgery of abdomen and thorax. ${ }^{3}$

Here, a case has been presented that used ESP block for pain management due to traumatic multiple rib fractures and prevented pulmonary complications with ESP block in an ICU.

Correspondence to: Dr. Canan Gursoy, Division of Intensive

Care Unit, Department of Anesthesiology and Reanimation,

Mugla Sitki Koçman University Training and Research Hospital,

Mugla, Turkey

E-mail: gursoycanan@yahoo.com

Received: March 22, 2019; Revised: July 05, 2019;

Accepted: August 22, 2019

\section{CASE REPORT}

The patient was a 34-year male, who had suffered road traffic accident. He had sustained fractures of left distal ulnar bone and $3^{\text {rd }}-6^{\text {th }}$ posterior ribs on the left side without a pneumothorax (Figure 1). He used parenteral analgesics (tramadol $300 \mathrm{mg}$ and paracetamol $3000 \mathrm{mg}$ per day) for thoracic pain during first two days, but the pain management was insufficient. On second day after accident, he had pulmonary failure associated with left lobar atelectasis because of ineffective cough effort and inadequate depth of breathing due to severe pain. When admitted to ICU, the patient was tachycardic and tachypnic. His oxygen saturation was $80 \% . \mathrm{PaO}_{2}$ $/ \mathrm{FIO}_{2}$ ratio and other data are shown in Table I. When evaluated with visual analogue scale (VAS), it was $6 / 10$ and $8 / 10$, respectively at rest and cough despite of having used parenteral analgesics. His respiration was supported by non-invasive ventilation (NIV) immediately. Regional anaesthesia -ESP block- for pain manangement in ICU was planned. The patient was placed in the

Table I: Parameters before and 6 hours after ESP block.

\begin{tabular}{l|c|c}
\hline & Before ESP & After ESP \\
\hline VAS rest & $6 / 10$ & $3 / 10$ \\
VAS cough & $8 / 10$ & $4 / 10$ \\
Heart rate (bpm) & 123 & 91 \\
Systolic blood pressure $(\mathrm{mmHg})$ & 158 & 98 \\
Diastolic blood pressure $(\mathrm{mmHg})$ & 123 & 73 \\
Frequency & 34 & 21 \\
Tidal volume $(\mathrm{ml})$ & 280 & 410 \\
$\mathrm{PaO}_{2} / \mathrm{FIO}_{2}$ & 101 & 163 \\
\hline
\end{tabular}

VAS = Visual analogue scale; $E S P=$ Erector spinae plane block. 


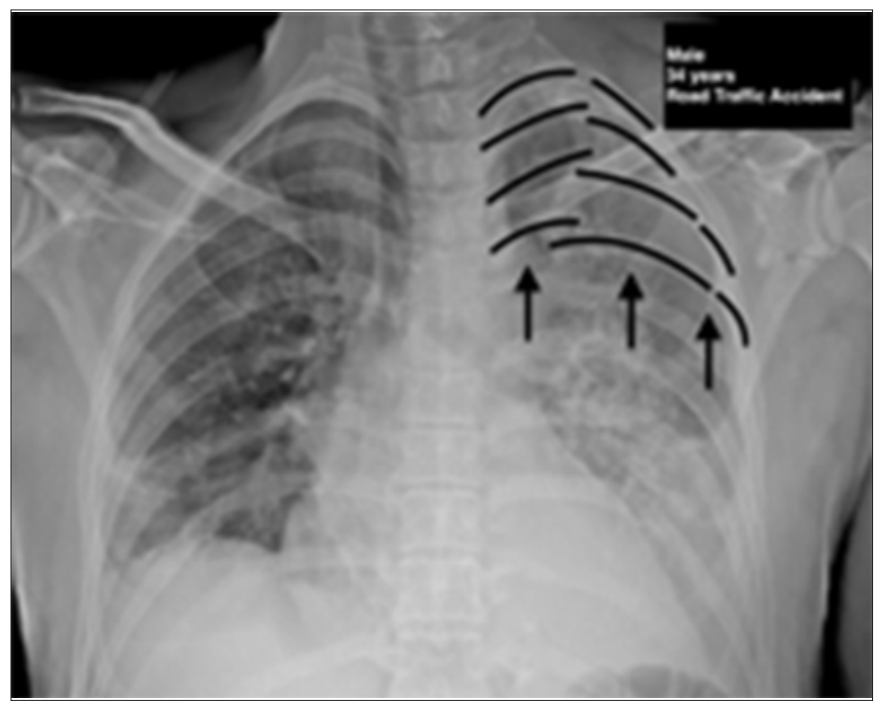

Figure 1: Chest X-ray (PA view) multiple rib fractures (arrows).

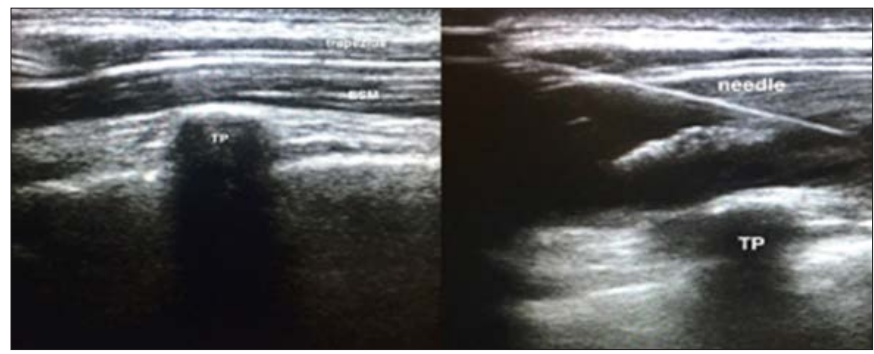

Figure 2: Ultrasound images of ESP Block.

sits position and ESP block guided with ultrasound was performed at the T4 level with $20 \mathrm{ml}$ of $0.25 \%$ bupivacaine (Figure 2). Catheter could not be placed because of non-availability of catheter. Only paracetamol (1000 mg per day) was administrated for relief of pain due to ulnar fracture.

Thirty minutes later, the patient reported control of thoracic pain. VAS was $1 / 10$. The patient began to breathe deeply and regularly. Tachycardia and tachypnea were decreased. Six hours after ESP block, $\mathrm{PaO}_{2} / \mathrm{FIO}_{2}$ ratio was dramtically improved (Table I). VAS was 3-4/10. Despite this level of pain, the patient was performing the necessary pulmonary exercises. So we did not approve invasive technique and administrated paracetamol with the same dose. Patients was weaned off from NIV support within 24 hours. 48 hours after ESP block, the patient was discharged from ICU to orthopedics department for fixation of ulnar fracture.

\section{DISCUSSION}

Pain management plays a key role in the treatment of traumatic multiple rib fractures. Conventional regional techniques such as thoracic epidural, thoracic paravertebral, intercostal and intrapleural block are most commonly used techniques for pain management. ${ }^{2}$ But these techniques, particularly epidural analgesia and paravertebral block, have limited use in some cases due to administration of anticoagulant therapy, multiple bone fractures or in patients who cannot be optimally positioned. ${ }^{3}$

ESP block, described by Altiparmark et al., is a novel myofascial plane block recently introduced into clinical practice. 4 It is simple to perform the block because the key land-marks of tip of transverse processes and erector spinae muscle are easily visualised on ultrasound. 3,5 When local anaesthetic is injected in this plane, initially the dorsal rami are blocked, later local anaesthetic spreads anteriorly and cephalocaudally in plane, which leads to ventral rami and intercostal nerves block. Cephalocaudal spread also provides anaesthesia to at least three segments, above; and four segments, below to injection site. It results in extensive thoracic anaestehesia with a single injection. ${ }^{3}$ This also allows to treat patients more easily in ICU. But the optimum dose and concentration of local anesthetic drug is unknown.4 In addition, ESP block provides effective analgesia starting within minutes. It has the potential to become vital analgesic modality for rib fractures. Furthermore, ESP block avoids the complications of conventional techniques such as hypotension, vascular puncture, local anaesthetic toxicity, pneumothorax and parenteral analgesic adverse effecs.5,6

In conclusion, in ICU, ESP block should be used more frequently for the treatment of pain in case of rib fractures with its easy application and rapid effects. It allows the patients to cough, breath deeply; and decreases the duration length of mechanical ventilation.

\section{PATIENT'S CONSENT:}

Patient provided both oral and written consent for the information gathering and publication of his case.

\section{CONFLICT OF INTEREST:}

Authors declared no conflict of interest.

\section{AUTHORS' CONTRIBUTION:}

CG: Designing, writing of manuscript, literature review. YK: Performed ESP block, literature review.

SGD: Designing, literature review.

\section{REFERENCES}

1. Carrie C, Stecken L, Cayrol E, Cottenceau V, Petit L, Revel P, et al. Bundle of care for blunt chest trauma patients improves analgesia but increases rate of intensive care unit admission: A retrospective case-control study. Anaesth Crit Care Pain Med 2018; 37:211-5.

2. Peek J, Smeeing DPJ, Hietbrink F, Houwert RM, Marsman M, de Jong MB. Comparison of analgesic interventions for traumatic rib fractures: A systematic review and meta-analysis. Eur $J$ Trauma Emerg Surg 2019; 45:597-622.

3. Thiruvenkatarajan V, Cruz Eng H, Adhikary S Das. An update on regional analgesia for rib fractures. Curr Opin Anaesthesiol 2018; 31:601-7. 
4. Altiparmak B, Korkmaz Toker M, Uysal Al, Turan M, Gümüs Demirbilek S. Comparison of the effects of modified pectoral nerve block and erector spinae plane block on postoperative opioid consumption and pain scores of patients after radical mastectomy surgery: A prospective, randomized, controlled trial. J Clin Anesth 2019; 54:61-5.

5. Jain K, Jaiswal V, Puri A. Erector spinae plane block: Relatively new block on horizon with a wide spectrum of applications: A case series. Indian J Anaesth 2018; 62:809-13.

6. Nandhakumar A, Nair A, Bharath VK, Kalingarayar S, Ramaswamy BP, Dhatchinamoorthi D. Erector spinae plane block may aid weaning from mechanical ventilation in patients with multible rib fractures: Case report of two cases. Indian $J$ Anaesth 2018; 62:139-41.

......... 